\title{
3D Printed Moulds Encompassing Carbon Composite Electrodes to Conduct Multi-Site Monitoring in the Entire Colon
}

\author{
Nirav Patel ${ }^{1}$, Aidan Fagan-Murphy ${ }^{1}$, Derek Covill ${ }^{3}$ and Bhavik Anil Patel ${ }^{1,2^{*}}$ \\ ${ }^{1}$ School of Pharmacy and Biomolecular Sciences, ${ }^{2}$ Centre for Stress and Age-related diseases \& \\ ${ }^{3}$ School of Computing, Engineering and Mathematics, University of Brighton, UK \\ ${ }^{*}$ Correspondence should be addressed to Dr Bhavik Patel; Email: $\underline{\text { b.a.patel@brighton.ac.uk; }}$ \\ Tel: $+44(0) 1273642418$
}




\section{ABSTRACT}

The activity of the colon is regulated by chemical signalling, of which serotonin (5HT) is a key transmitter. Monitoring of mucosal 5-HT overflow has been achieved to date using microelectrodes on small segment of colonic tissue, however little is known if such measurements are reflective with regards to $5-\mathrm{HT}$ signalling from the entire colon. This study focused on developing an electrochemical array device that could be utilised to conduct multi-site measurements of 5-HT overflow from the entire colon. A 3D printed mould was fabricated that could house 6 multi-wall carbon nanotube composite electrodes and provide a fixed distance between the electrodes and the tissue along the entire length of the colon. The electrodes were assessed for sensitivity, stability and crosstalk before conducting in vitro measurements using colons obtained from 6 and 24 month old mice. As composite electrodes can have a high degree of variability, normalisation factors were required between electrodes for a given array. The device had the sensitivity and stability required for 5-HT measurements from intestinal tissue. Regio-specific changes in 5-HT overflow were observed with age, where increases in 5-HT overflow were observed in the distal colon due to an impairment/loss in the serotonin transporter (SERT). Our strategy can be utilised to develop arrays of varying sizes and geometries which can offer practical solutions for large scale tissue measurements.

\section{Keywords}

Multi-site, spatial, enterochromaffin cell, mucosa, colon, serotonin, serotonin transporter (SERT), ageing 


\section{INTRODUCTION}

The colon is an important organ that is responsible for the absorption of water and processing of waste products from the body for defection. The inner most layer of the colon, known as the mucosa contains enterochromaffin (EC) cells, which release serotonin $(5-\mathrm{HT})$ following luminal mechanical or chemical stimulation ${ }^{1}$. The $5-\mathrm{HT}$ stimulates intrinsic primary afferent neurons (IPANs) which drive a cascade of neurons within the myenteric plexus that drive the smooth muscle to regulate motility 2,3. Most digestive disorders, such as inflammatory bowel disorders (IBD) or chronic constipation occur within the colon ${ }^{4,5}$ and numerous studies have shown $5-\mathrm{HT}$ signalling is altered in these conditions using very small segments of the colon or ileum ${ }^{6-9}$. Such changes can have implications toward the dysfunction observed as 5$\mathrm{HT}$ is important in the regulation of motility. However, it is not well known if these small segments of tissues provide a realistic reflection on $5-\mathrm{HT}$ signalling within the entire organ.

Electrochemical studies using carbon fibre or boron doped diamond microelectrodes have observed changes in 5-HT signalling with IBD, obesity and ageing on small segments (usually $1 \mathrm{~cm}^{2}$ ) of the colon and colon ${ }^{6,10-13}$. Often the results of these studies are interpreted to explain the functional changes observed. Electrochemical recording in such small areas of the colon have provided valuable insight into signalling changes but significantly underestimate the impact of such biological conditions on the behaviour of the entire colon.

Multi-site or spatial monitoring is a means of monitoring signalling molecules over various anatomical regions of cells and tissues. Numerous studies have been conducted on single cells and in brain regions in vivo ${ }^{14-17}$. Such studies have 
provided insight into how transmitter signalling occurs in distinct locations and therefore has improved our understanding on transmitter signalling mechanisms. These measurements have been conducted using electrochemical array devices that contain electrodes that are separated by less than $1 \mathrm{~mm}$ and have micrometre dimensions. These dimensions are attractive for single cell studies or tissue regions where penetrative measurements are conducted, as the devices have appropriate dimensions to the biological environment and are less invasive. However, such approaches provide limited when multi-site recording is needed to be conducted over larger tissue surface areas $\left(>1 \mathrm{~cm}^{2}\right)$.

At present planar electrode arrays are often fabricated using complex approaches involving lithography or screen printing, which are very expensive if utilised to fabricate large dimensions $(>2 \mathrm{~cm})$. Therefore, to conduct multi-site measurements in bigger dimensions, fabrication strategies are needed to ensure devices are analytically robust, reproducible and inexpensive. One option is the use of composite based sensors, which provide the ability to create electrodes of varying carbonbased materials that can be moulded and shaped into various geometries ${ }^{18-20}$. We have previously shown that multi-wall carbon nanotube (MWCNT) composite electrodes can show comparable performance to boron-doped diamond (BDD) electrodes for the detection of $5-\mathrm{HT}^{21,22}$.

Within this study, we have developed a $3 D$ printed mould that can house MWCNT composite sensors and therefore can be utilised to develop array based electrodes in dimension suitable for measurements of large sections of tissues. We fully characterised the array device to understand the influence of electrode crosstalk and electrode fouling. Studies were carried out using the entire colon from young and old aged murine to understand changes in mucosal 5-HT signalling. 


\section{EXPERIMENTAL SECTION}

\section{Fabrication of the 3D printed mould to encompass the carbon composite electrodes}

The 3D printed mould was created to encompass the electrodes and provide a uniform distance between the electrodes and the tissue along the entire length of the colon. The mould was designed using SolidWorks (Dassault Systèmes) and a drawing highlighting the key dimensions of the mould as printed is shown in Figure 1A. The device was $50 \mathrm{~mm}$ long and $7 \mathrm{~mm}$ wide to span the entire length of the murine colon. The mould contained six evenly spaced slots to encompass the electrodes and these were $8 \mathrm{~mm}$ apart. The square slots for the electrodes were 3.6 $\times 3.6 \times 2 \mathrm{~mm}$, and the centre of the electrode slot contained a $1.6 \mathrm{~mm}$ diameter hole to feed an electrical wire through to make connection with the composite carbon electrode material. At the top of the mould, $1 \mathrm{~mm}$ vertical spacers were created above the level of the electrodes in-between the rectangular slots to ensure a consistent spacing between the electrode and the tissue, to reduce the prevalence of biofouling. The mould was 3D printed using a Replicator 2X (Makerbot Inc.) using Makerbot ABS plastic as shown in Figure 1B\&C.

To place the electrodes in the slots within the 3D mould, an insulated copper wire was fed through the back of the device and held in position using conductive silver epoxy. The rectangular slot was then packed with $15 \% \mathrm{w} / \mathrm{w}$ of MWCNT to $85 \%$ of epoxy resin/hardener mix (where epoxy resin to hardener ratio was $3: 1$, Robnor Resins Ltd. Swindon, Wilts, UK) to create the electrodes. The electrodes were left to cure for 48 hours at room temperature, initially ground down using fine sandpaper to 
the plane of the mould and then device was placed in an alumina slurry and gently polished in-between the raised features using a cut piece of polish pad to ensure they were completely level with the surface of the 3D printed mould (Figure 1B\&C).

A

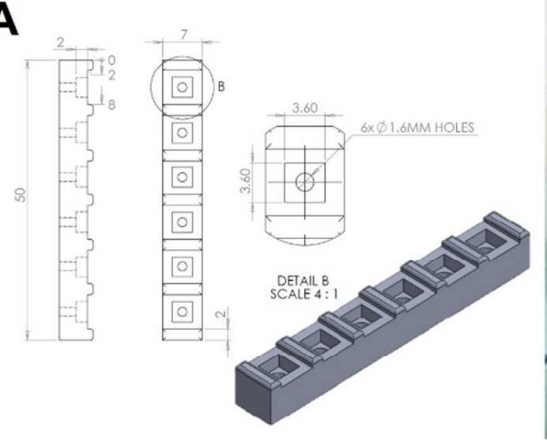

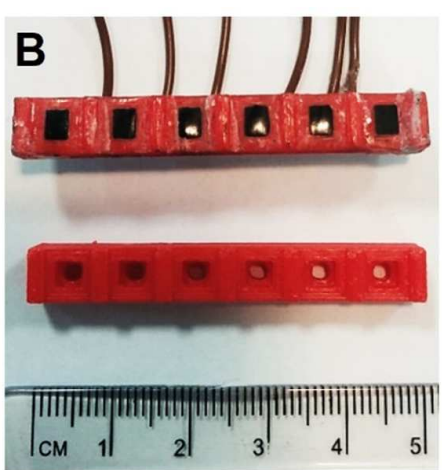

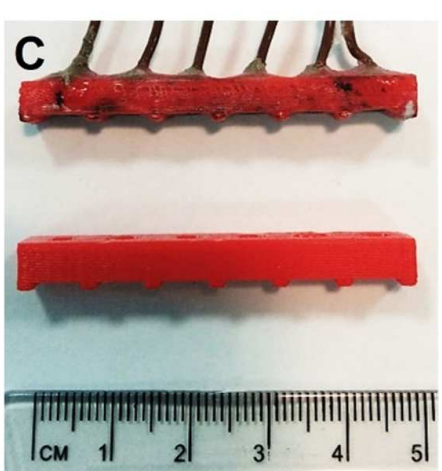

Figure 1: Fabrication of MWCNT carbon composite array. (A) key dimensions and 3D CAD model of the mould which was printed and used to encompass the electrodes. The final composite array device viewed from the top (B) and side (C).

\section{Characterisation studies}

All measurements were carried out using a 3-electrode system using a $\mathrm{Ag} \mid \mathrm{AgCl}$ reference electrode and Pt wire as the counter electrode. Recordings were obtained using $\mathrm{CH}$ Instruments 1030B multichannel potentiostat.

Each electrode within the array was tested in $1 \mathrm{mM}$ potassium ferrocyanide in $1 \mathrm{M}$ $\mathrm{KCl}$ to obtain a response which could be utilised to normalise each electrode response. Normalisation factors were used to assess if the device could accurately show the same current response for $5 \mu \mathrm{M} 5-\mathrm{HT}$ in $0.1 \mathrm{M}$ PBS. The current response of various solution in the concentration range of $1-20 \mu \mathrm{M}$ were carried out to determine the sensitivity and limit of detection of the electrodes.

The crosstalk between adjacent electrodes was assessed using $1 \mathrm{mM}$ potassium ferrocyanide in $1 \mathrm{M} \mathrm{KCl}$, where the detection electrode was subjected to a triangle 
waveform $\left(-0.2\right.$ to $0.8 \mathrm{~V}$ at $\left.0.1 \mathrm{~V} \mathrm{~s}^{-1}\right)$, whilst the adjacent electrodes were run amperometrically at $0.8 \mathrm{~V}$. Measurements were carried out at a flow rate of 2 $\mathrm{mL} / \mathrm{min}$. To mimic a tissue simulated environment, the array was covered with a layer of stretched parafilm.

The performance of the electrodes to biofouling and post in vitro measurements were assessed. For biofouling studies, measurements were carried out in $1 \mathrm{mM}$ potassium ferrocyanide in $1 \mathrm{M} \mathrm{KCl}$ after exposure to $0.5 \% \mathrm{w} / \mathrm{v}$ mucin for 1 and 2 hours. Measurements were also carried out to understand the degree of sensor biofouling following tissue measurements using $1 \mathrm{mM}$ potassium ferrocyanide in $1 \mathrm{M}$ $\mathrm{KCl}$. Typically, measurements for each tissue were less than 1 hour in duration. For all measurements in ferricyanide, cyclic voltammetry was utilised using a potential window of -0.2 to $0.8 \mathrm{~V}$ at $0.1 \mathrm{~V} \mathrm{~s}^{-1}$ scan rate.

\section{Studies in the entire colon}

All procedures were carried out according to U.K. Home Office regulations and were approved by the University of Brighton Ethics Committee. Male C57BL/6J mice were obtained from Harlan UK at 8 weeks of age and housed in individual ventilated cages in groups of 3-4, under barrier-reared conditions until required. Animals were maintained at $19.0 \pm 1{ }^{\circ} \mathrm{C}, 55 \%$ humidity and fed on a maintenance diet (irradiated RM1 (E) 801002 chow, Special Diet Services) and had free access to irradiated water. Animals aged either 6 or 24 months were euthanized in $\mathrm{CO}_{2}$, followed by cervical decapitation and the entire colon was harvested and placed in ice cold oxygenated $\left(95 \% \mathrm{O}_{2}\right.$ and $\left.5 \% \mathrm{CO}_{2}\right)$ Krebs' buffer solution, $\mathrm{pH} 7.4(117 \mathrm{mM} \mathrm{NaCl}, 4.7$ 
$\mathrm{mM} \mathrm{KCl}, 2.5 \mathrm{mM} \mathrm{CaCl}_{2}, 1.2 \mathrm{mM} \mathrm{MgCl}_{2}, 1.2 \mathrm{mM} \mathrm{NaH}_{2} \mathrm{PO}_{4}, 25 \mathrm{mM} \mathrm{NaHCO}_{3}$ and 11 $\mathrm{mM}$ glucose) prior to experiments.

The 3D printed mould encompassing the carbon composite electrodes was embedded in a Sylgard®-(Dow Corning, UK) lined Teflon recording chamber. The colon was opened along its mesenteric border and placed on the mould with the mucosal layer facing the electrodes. The tissue was stretched around the mould and pinned to the adjacent Sylgard, so that the tissue-electrode distance would be fixed at $1 \mathrm{~mm}$ over all electrodes. The Teflon recording chamber was perfused with warm $\left(37^{\circ} \mathrm{C}\right)$ Krebs solution at a flow rate of $2 \mathrm{~mL} / \mathrm{min}$. Tissues were perfused for 30 minutes prior to commencing a series of measurements. To identify the presence of 5-HT, differential pulse voltammograms were recorded from 0 to $1 \mathrm{~V}$, and for measurements of 5-HT overflow, amperometric recordings were carried out on all electrodes for a duration of $30 \mathrm{~s}$ at $0.6 \mathrm{~V}$ vs. $\mathrm{Ag} \mid \mathrm{AgCl}$ reference electrode. Measurements were carried out to compare 5-HT overflow between 3 and 24-monthold animals in the presence and absence of SERT blocker fluoxetine and 5-HT4 agonist prucalopride.

\section{Data Analysis}

To obtain similar current profiles for all electrodes, the response of $1 \mathrm{mM}$ ferrocyanide was obtained and used to normalise currents from one electrode to another. Conversion factors were obtained for each electrode within a single array device and the electrode with the smallest responses was utilised to normalise all other electrodes for all investigations conducted within the study. 
For all characterisation studies, oxidation peak current amplitudes were obtained and compared for each of the variables investigated. Data was presented as mean \pm st.dev., where statistical analysis was carried out using either a Students t-test or one-way ANOVA. For biological studies on the entire colon, the difference in the mean current amplitude observed in bulk media and in the presence of tissue was recorded between $35-40 \mathrm{~s}$ at each electrode. This was then plotted to understand the influence of age and pharmacological agents on serotonin overflow and statistically interpreted using a two-way ANOVA.

\section{RESULTS AND DISCUSSION}

\section{Device characterisation and electrode normalisation}

Each of the composite MWCNT electrodes was characterised on individual devices using $1 \mathrm{mM}$ ferrocyanide. Although carbon composite electrodes offer numerous benefits, such as allowing the ability to fabricate varying electrode geometries, they often suffer from high variability from electrode to electrode. Figure 2 shows the variation in the ferrocyanide voltammetric response on electrodes 1-6 for a given device. The mean coefficient of variation for all electrodes on a single electrode array was $8.7 \pm 2.1 \%$ ( $n=6$ arrays). A representative trace showing how the current amplitude of ferrocyanide varied on individual electrodes on an array is shown in Figure 2A. This observed variation can result in inaccurate estimations of the responses during biological recordings between electrode to electrode and from device to device. Therefore, the current amplitude of ferrocyanide for the electrode with the smallest current amplitude on the device was utilised to normalise the responses of all other electrodes. When this approach was utilised as shown in 
Figure 2B, greater consistency in the ferrocyanide voltammograms was observed. Normalisation parameters based on the ferricyanide response were applied for the detection of $5 \mu \mathrm{M} 5-\mathrm{HT}$ at each of the electrodes (Figure 2C). The coefficient of variation for all electrodes on various arrays was reduced to $2.8 \pm 1.1 \%$ ( $n=6$ arrays) for the detection of 5-HT. Application of the normalisation factors is important when using composite electrodes in array based devices to reduce error in signal detection between electrodes.
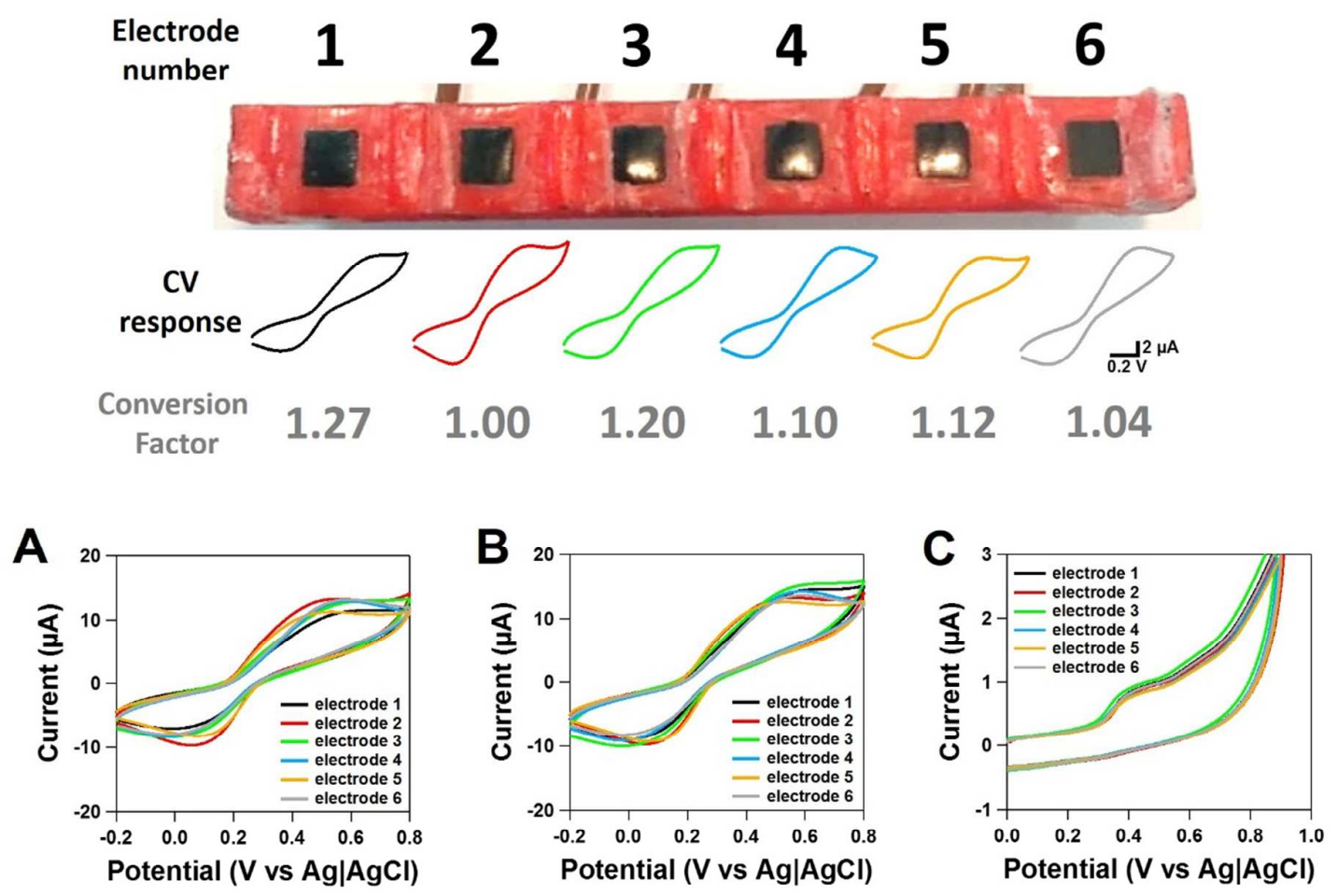

Figure 2. Electrochemical responses on the MWCNT composite array and responses following normalisation. Due to the composite material, conversion factors are utilised to normalises responses between electrodes, which are shown for a representive device. Response of $1 \mathrm{mM}$ potassium ferrocyanide in $1 \mathrm{M} \mathrm{KCl}$ before $(A)$ and after normalisation (B). (C) shows response for $1 \mu \mathrm{M}$ serotonin in 0.1 M PBS following normalisation.

The performance of the electrode arrays for the detection of $5-\mathrm{HT}$ at physiological levels $(1-20 \mu \mathrm{M})$ was evaluated, where the limit of detection for the electrodes on a 
given array was $650 \mathrm{nM}$ and the sensitivity was $102 \mathrm{nA} \mu \mathrm{M}^{-1}$ (n=5 devices). The sensitivity observed seemed fit for purpose for measurement of 5-HT from colon tissue based on previous studies ${ }^{9}$.

\section{Understanding electrode crosstalk}

Crosstalk measurements were carried out to understand the extent of overlap in diffusional pathways between adjacent electrodes. Similar studies have been conducted on electrochemical array devices ${ }^{16}$, where electrodes were shown to have overlapping diffusional pathways and therefore decreased current responses. Measurements were carried out in $1 \mathrm{mM}$ ferricyanide, where the electrode investigated for crosstalk was run using a voltammetric waveform, whilst the adjacent electrodes were run amperometric at $0.8 \mathrm{~V}$, which was sufficient to oxidise ferrocyanide. For crosstalk measurements, all experiments were carried out in flowing solution of ferrocyanide. Measurements were conducted where the array device was uncovered (Figure $\mathbf{3 A}$ ) and then covered with a stretched layer of parafilm to mimic a tissue placed on the device (Figure 3B).

Figure $3 \mathrm{C}$ shows a representative experimental trace, showing the voltammetric response of the electrode investigated for crosstalk interference with and without adjacent electrodes being run amperometrically at $0.8 \mathrm{~V}$. The cyclic voltammograms over time can show the presence of the oxidation peak which was measured in the presence and absence of adjacent electrodes being run amperometrically. The difference in the oxidation peak current when adjacent electrode were switch on or off was used to indicate the degree of crosstalk. The overall data from crosstalk studies are shown in Figure 3D. There was a significant decrease in the 
ferrocyanide current of a given electrode when adjacent electrodes are were run at $0.8 \mathrm{~V}$ when compared to the current response of the electrode run when adjacent electrodes were not active $((p<0.05, n=4)$. This is suggestive that there was overlap in the diffusional pathways between adjacent electrodes in the MWCNT composite electrode arrays, resulting in a reduction in the electrode response. This may also be due to the convention utilised in the measurement to replicate the environment in which tissue measurements are conducted. When the crosstalk study was conducted in a tissue stimulated environment, there was no difference in the response of the electrode with and without adjacent electrodes run amperometrically to oxidise ferricyanide. (Figure 3D). This finding suggests that the parafilm film, which acts to mimic the tissue, creates a closed environment above the electrode, by the parafilm forming a seal with the raised elevations in-between the electrodes on the 3D printed device. These closed environments between the electrodes which can be observed in the photograph in Figure 3B provide the means to overcome issues with crosstalk by compartmentalising the electrodes to the localised tissue above and potentially enhance the sensitivity of the response.
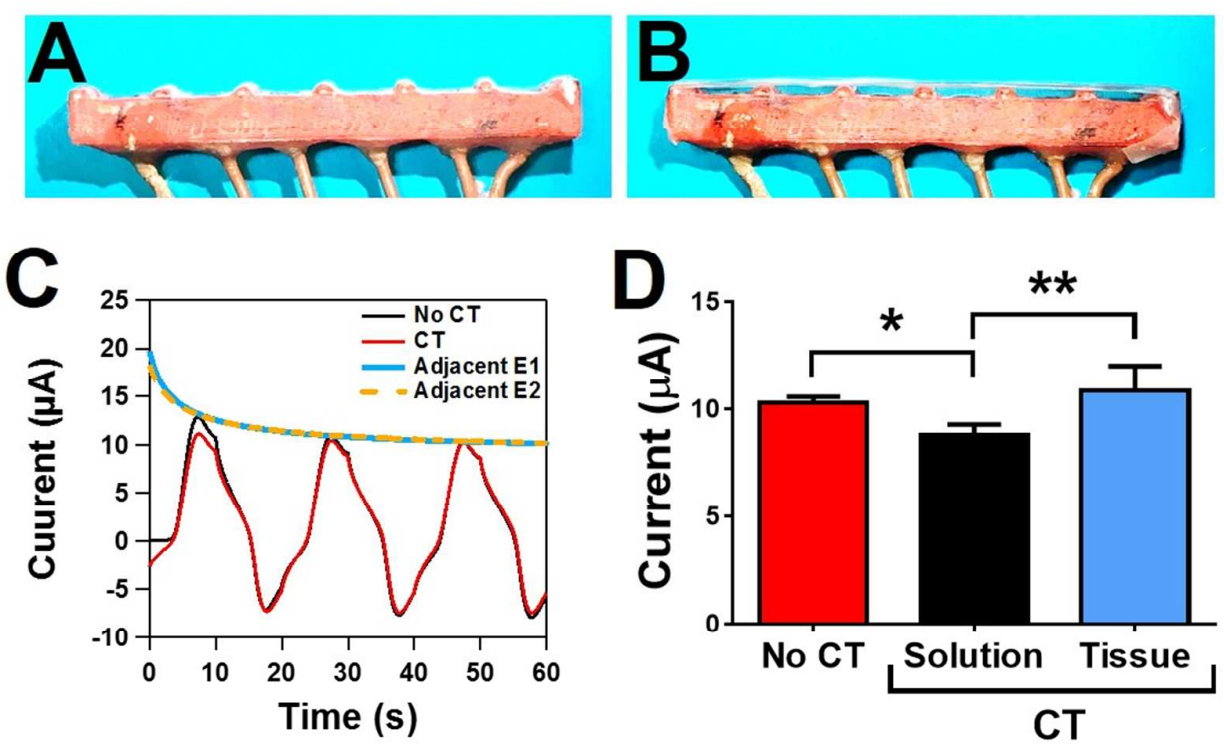
Figure 3. Investigation of crosstalk (CT) between adjacent electrodes. The experimental set up was conducted with the electrodes exposed to the flowing solution $(\mathrm{A})$ and when covered with parafilm to simulate tissue measurements (B). (C) Cyclic voltammetry $\left(0.1 \mathrm{Vs}^{-1}\right)$ and amperometric studies $(0.8 \mathrm{~V})$ in $1 \mathrm{mM}$ potassium ferrocyanide were utilised to investigate crosstalk. Where E1 and E2 indicate the electrodes adjacent to the electrode investigated for crosstalk interference. The oxidative peak current when the adjacent electrode were run amperometrically was compared to that when the adjacent electrode where not active. (D) The overall data from multiple studies in flowing solution and tissue environment. Data shown as mean \pm st.dev, $n=4,{ }^{*} p<0.05$.

\section{Investigation of electrode fouling}

Electroanalytical measurements conducted from gastrointestinal tissue pose significant problems from biofouling due to the release of mucins from the mucosa, which act to lubricate the bowel and from oxidative by-products of $5-\mathrm{HT}$, that have been shown to foul carbon electrode surfaces ${ }^{23,24}$. Figure 4 shows an investigation into the stability of the MWCNT carbon composite electrodes in mucin and following isolated colon measurements. Initially, measurements were carried in ferrocyanide, where the current response was compared pre- and post-exposure to $5 \% \mathrm{v} / \mathrm{w}$ mucin for 1 or 2 hours (Figure 4A). When looking at the current amplitude of ferrocyanide following mucin exposure on individual electrodes, there was a significant decrease in the current after $1(p<0.05$ for only electrode 2 and $6, n=6)$ and 2 hours $(p<0.01$ for electrodes 4 and $5 ; p<0.001$ for electrodes $1,2,3$ and $6 ; n=6$, Figure 4B). The overall pooled data for all electrodes on various arrays showed a significant decrease after 1 hour and 2 hours $(p<0.01, n=6$, Figure $4 C)$. There was also a significant decrease in the current response observed between 1 and 2 hours $(p<0.05, n=6)$.

These findings are consistent with other studies that have shown that carbon based electrodes are prone to biofouling ${ }^{25,26}$. Studies have highlighted that carbon 
nanotubes provide a degree of resistance to fouling compared to conventionally used carbon materials such as glassy carbon and carbon fibre ${ }^{27}$. The approximate $10 \%$ reduction in the current after 2 hours in mucin still seems a satisfactory performance of the MWCNT material as previous studies have shown glassy carbon and boron-doped diamond are more prone to fouling in $\operatorname{mucin}^{23}$.

Studies were also conducted to understand the degree of fouling that occurred post measurements with the isolated colon. During such studies, the ferrocyanide response was measured before and after a biological measurement with the isolated colon for a duration of 1 hour, where recording of 5-HT overflow was repeated between 6-8 times for a duration of $30 \mathrm{~s}$ each. A representative cyclic voltammogram of ferrocyanide before and after tissue recordings is shown in Figure 4D, where the amplitude of the ferrocyanide response was not altered but there were changes in the shape of the response, suggesting some degree of fouling.

The response on individual electrodes are shown in Figure 4E, where small reductions in the current were observed but were not significantly different to the ferrocyanide response obtained prior to biological measurements. The overall pooled data for all electrodes on various arrays showed no significant decrease in the current response post biological measurement $(p=0.11, n=6)$, therefore suggestive that for our measurement protocol with the isolated colon, the response of the electrode array was stable.

Overall the degree of fouling is significantly less than that observed in microelectrodes, which is most likely due to the larger size of the electrode. The difference between the two measurement is interesting, as no fouling is observed following measurement from the isolated colon, where the electrodes were exposed 
to oxidative by-products of $5-\mathrm{HT}$ oxidation and mucins released from the mucosa.

This was not anticipated as significant fouling was observed on the electrodes when measurements were carried out in mucins. The most likely explanation of these findings, is that the measurement environment was varied, as studies in tissues were conducted with convection of the physiological buffer to keep the tissue viable and therefore providing convention to reduce the rate of electrode fouling compared to measurements in static solution.
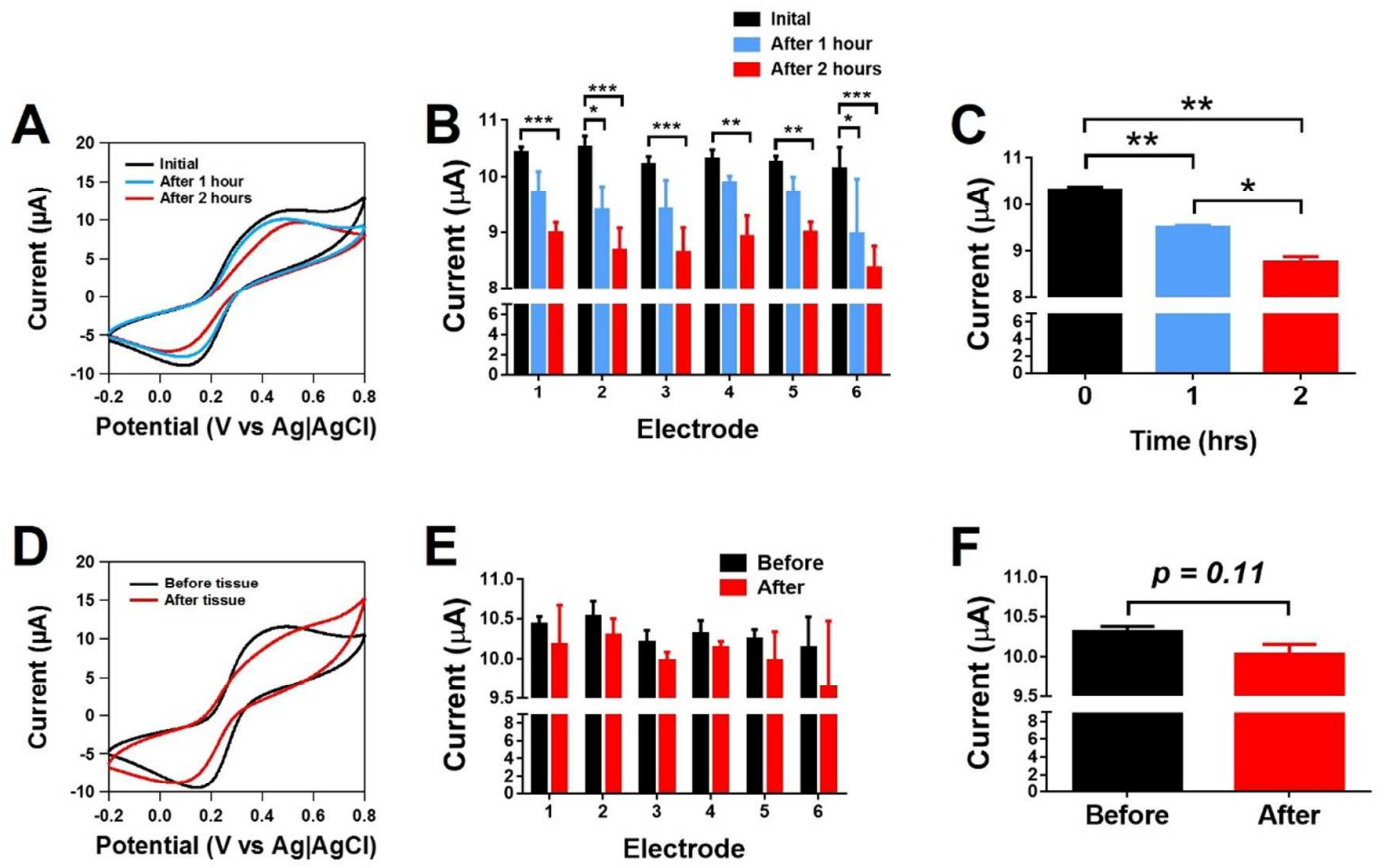

Figure 4. Fouling studies on the MWCNT composite array. (A) potassium ferricyanide responses before and after measurements in $0.5 \% \mathrm{w} / \mathrm{v}$ mucin, where (B) shows individual electrode responses and (C) shows overall response from multiple arrays. (D) potassium ferricyanide response before and after measurements from the isolated murine colon for 1 hour. (E) Responses of individual electrodes before and after tissue measurements and (F) the overall responses following in vitro measurements. Data shown as mean \pm st.dev, ${ }^{*} p<0.05{ }^{* *} p<0.01$ and ${ }^{* * *} p<0.001$

Investigation of 5-HT release in the entire colon with age 
Measurements were carried out using the entire colon, where the mucosal layer was placed facing the electrodes and the tissue was stretched and pinned to the width of the device as shown in Figure 5A. Electrode 1 was located closest to the proximal colon and electrode 6 was located closest to the distal colon. For measurements, recordings were carried out using differential pulse voltammetry, where oxidation currents for $5-\mathrm{HT}$ were observed on all 6 electrodes at $0.35 \mathrm{~V}$. When recordings were carried out in colon tissue where the mucosal tissue was removed, no oxidation peak was observed (Figure 5B). The amplitude of the current observed between 6 and 24-month-old animals was compared at each electrode (Figure 5C). In the 6 month old animal, there was a significant increase in the current observed at electrode 3 and 4 when compared to electrode 1,2 and $6(p<0.01, n=5)$. This is an unusual finding as most studies have indicated that 5-HT release decreases through the digestive tract from oral to anal, however this finding suggests there is a significantly higher overflow of $5-\mathrm{HT}$ in the mid-point of the colon in the murine. This region-specific variation in 5-HT might be associated with the colon containing a junction driven by chemical signalling that acts to prevent continuously motility from proximal to distal colon. There was a significant increase in the overflow of $5-\mathrm{HT}$ at electrode 5 and $6(p<0.001, n=5$, Figure 5C) in 24 month old animals when compared to 6 month old animals. This increase in 5 -HT overflow is most likely to be due to elevated release and/or loss in the function of the SERT with age. Similar observations were observed in isolated 3 and 24 month old segments of murine distal colon using BDD microelectrode ${ }^{13}$.

To understand what could explain the changes observed with age, we conducted studies in the presence of $1 \mu \mathrm{M}$ fluoxetine, which acts to block SERT and therefore elevates 5-HT overflow. Figure 5D shows the influence of fluoxetine in 6 month old 
animals, where an increase in the signal was observed at electrode 1, 2, 5 and 6 $(p<0.001, n=5)$. Although modest increases were observed at electrode 3 and 4 , these were not significant. These findings would indicate that SERT was functional in both distal and proximal regions of the colon in 6 month old animals. With no significant increase for fluoxetine observed at electrode $3 \& 4$, is suggestive that the transporter density/activity was limited compared to the adjacent regions. In 24 month old animals, only a significant increase in the current was observed in the presence of fluoxetine at electrodes 1 and $2(p<0.001, n=5$, Figure $5 E)$. This is suggestive that the increased $5-\mathrm{HT}$ observed in 24 month animals was most likely due to impaired/loss in the 5-HT transporter with age in the distal colon. Such increases in 5-HT overflow are most likely to desensitise the 5-HT receptors on the intrinsic primary afferent neurons, resulting in reduced motility. We have through motility studies observed decreases in motility within only the distal region, which is most likely to be associated with the impaired 5-HT signalling in the distal colon when compared to the proximal colon ${ }^{28}$.

We have previously shown the $5-\mathrm{HT}_{4}$ receptor on the C57BL6 murine EC cell to function as an inhibitory autoreceptor which can be utilised to reduce the overflow of $5-\mathrm{HT}^{12}$. Therefore, we utilised selective $5-\mathrm{HT} 4$ agonist prucalopride to understand if this could reduce the5-HT overflow in aged mice. In 6 month old animals, we observed a decrease in 5-HT overflow at electrodes 3 and $4(p<0.001, n=5$, Figure 5F). Similarly decreases in 5-HT overflow were observed in 24 month old animals at electrodes $3,4(p<0.001)$ and $5(p<0.01, n=5$, Figure $5 E)$. These findings suggest that prucalopride might be a useful therapeutic agent in supressing the $5-\mathrm{HT}$ overflow, which may be useful in reducing age related increase in $5-\mathrm{HT}$ release or those observed in inflammatory bowel conditions, which lead to dysmotility. 

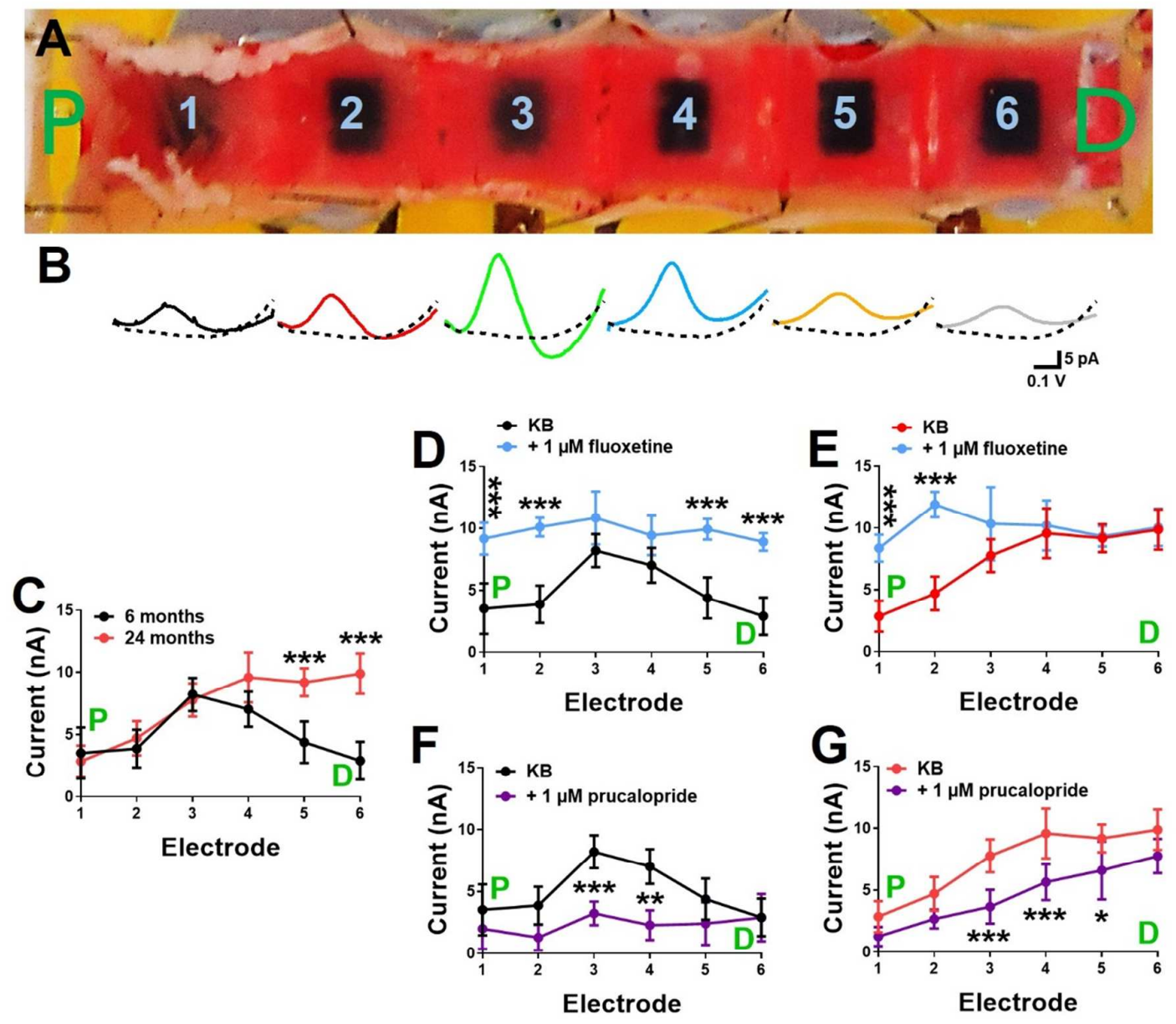

Figure 5. Studies from entire murine colon. (A) Photograph showing the entire colon positioned over the electrode array, where $P$ is proximal and $D$ is distal region. (B) shows a representative DPV responses in the presence (solid line) and absence (dashed line) of the mucosal layer of the colon for each electrode. (C) responses from 6 and 24 month old animals. (D) responses from 6 months and (E) 24 month animals in the presence of SERT inhibitor fluoxetine. (F) responses from 6 months old and $(G) 24$ month old animals in the presence of $5-\mathrm{HT}_{4}$ agonist prucalopride. Data shown as mean $\pm \mathrm{st}$.dev, $\mathrm{n}=5,{ }^{* * *} \mathrm{p}<0.001$

\section{CONCLUSION}

We have created a 3D printed mould that can house carbon composite electrodes and be fabricated to any size and geometry to provide the means to conduct multisite measurements over large tissue regions. Our MWCNT composite sensors detected physiological levels of 5-HT present within the bowel and were not affected 
by fouling during tissue measurements. Using our array device, we observed regiospecific differences in 5-HT overflow within the colon, where elevated levels of 5-HT were only present in the distal colon. These changes were most likely due to a loss/impairment of SERT. Overall our device provided a valuable analytical tool to understand the changes in signalling within the bowel and how these may alter in specific condition in distinct regions.

\section{ACKNOWLEDGEMENTS}

The authors would like to thank Shire-Movetis, NV for supply of prucalopride utilised within this study. The authors would like to acknowledge the staff in the Bioresources centre for maintaining and handling of the animals utilised within this study.

\section{REFERENCES}

(1) Racke, K.; Reimann, A.; Schworer, H.; Kilbinger, H., Behav. Brain Res. 1995, 73, 83-87.

(2) Bertrand, P. P.; Bertrand, R. L., Autonomic neurosci. 2010, 153, 47-57.

(3) Mawe, G. M.; Hoffman, J. M., Nat Rev Gastroenterol Hepatol 2013, 10, 473-486.

(4) Costedio, M.; Hyman, N.; Mawe, G., Dis. Colon Rect. 2007, 50, 376-388.

(5) Mawe, G., Aliment. pharm. ther. 2006, 23, 1067-1076.

(6) Bertrand, P. P.; Barajas-Espinosa, A.; Neshat, S.; Bertrand, R. L.; Lomax, A. E., Am J Physiol Gastrointest Liver Physiol 2010, 298, G446-455. 
(7) Coates, M. D.; Mahoney, C. R.; Linden, D. R.; Sampson, J. E.; Chen, J.; Blaszyk, H.; Crowell, M. D.; Sharkey, K. A.; Gershon, M. D.; Mawe, G. M.; Moses, P. L., Gastroenterology 2004, 126, 1657-1664.

(8) Linden, D. R.; Chen, J.-X.; Gershon, M. D.; Sharkey, K. A.; Mawe, G. M., Am J Physiol Gastrointest Liver Physiol 2003, 285, G207-216.

(9) Patel, B. A., Neurogastroent. Motil. 2011, 23, 595-605.

(10) Bertrand, R. L.; Senadheera, S.; Markus, I.; Liu, L.; Howitt, L.; Chen, H.; Murphy, T. V.; Sandow, S. L.; Bertrand, P. P., Endocrinology 2011, 152, 36-47. (11) Nzakizwanayo, J.; Dedi, C.; Standen, G.; Macfarlane, W. M.; Patel, B. A.; Jones, B. V., Sci. Reports 2015, 5, 17324.

(12) Patel, B. A., Neurogastroent. Motil. 2016, 28, 914-923.

(13) Patel, B. A.; Fidalgo, S.; Wang, C.; Parmar, L.; Mandona, K.; Panossian, A.; Flint, M. S.; Ranson, R. N.; Saffrey, M. J.; Yeoman, M. S., Sci. Reports 2017, 7, 42754.

(14) Talauliker, P. M.; Price, D. A.; Burmeister, J. J.; Nagari, S.; Quintero, J. E.; Pomerleau, F.; Huettl, P.; Hastings, J. T.; Gerhardt, G. A., J Neurosci. Methods 2011, 198, 222-229.

(15) Lin, Y.; Trouillon, R.; Svensson, M. I.; Keighron, J. D.; Cans, A.-S.; Ewing, A. G., Anal. Chem. 2012, 84, 2949-2954.

(16) Zachek, M. K.; Park, J.; Takmakov, P.; Wightman, R. M.; McCarty, G. S., Analyst 2010, 135, 1556-1563.

(17) Zhang, B.; Adams, K. L.; Luber, S. J.; Eves, D. J.; Heien, M. L.; Ewing, A. G., Anal. Chem. 2008, 80, 1394-1400.

(18) O'Hare, D.; Macpherson, J. V.; Willows, A., Electrochem. Comm. 2002, 4, 245250. 
(19) Ramirez-Garcia, S.; Alegret, S.; Cespedes, F.; Forster, R. J., Analyst 2002, 127, 1512-1519.

(20) Ramírez-García, S.; Alegret, S.; Céspedes, F.; Forster, R. J., Anal. Chem. 2004, $76,503-512$.

(21) Fagan-Murphy, A.; Kataria, S.; Patel, B. A., J Solid State Electrochem 2016, 1-

8.

(22) Fagan-Murphy, A.; Patel, B. A., Electrochimica Acta 2014, 138, 392-399.

(23) Fagan-Murphy, A.; Watt, F.; Morgan, K. A.; Patel, B. A, J. Electroanal. Chem. $2012,684,1-5$

(24) Wrona, M. Z.; Dryhurst, G., J. Organic Chem. 1987, 52, 2817 -2825.

(25) Harreither, W.; Trouillon, R.; Poulin, P.; Neri, W.; Ewing, A. G.; Safina, G., Anal. Chem. 2013, 85, 7447-7453.

(26) Huffman, M. L.; Venton, B. J., Analyst 2009, 134, 18-24.

(27) Swamy, B. E. K.; Venton, B. J., Analyst 2007, 132, 876-884.

(28) Patel, B. A.; Patel, N.; Fidalgo, S.; Wang, C.; Ranson, R. N.; Saffrey, M. J.;

Yeoman, M. S., Experimental Gerontol. 2014, 53, 24-30. 
For TOC only

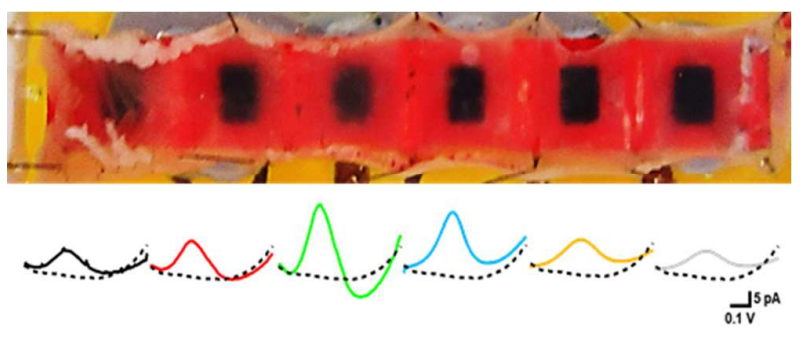

17 